\title{
Speckle tracking can detect subclinical myocardial dysfunction in rheumatoid arthritis patients
}

\author{
Benacka $\mathrm{O}^{1}$, Benacka $\mathrm{J}^{3}$, Blazicek $\mathrm{P}^{2}$, Belansky $\mathrm{M}^{2}$, Payer $\mathrm{J}^{1}$, Killinger $\mathrm{Z}^{1}$, Lietava $\mathrm{J}^{2}$ \\ 5th Department of Internal Medicine, Comenius University, Bratislava, Slovakia. \\ ondrejbenacka@gmail.com
}

\begin{abstract}
INTRODUCTION: Patients with rheumatoid arthritis (RA) have shorter life expectancy and their risk of cardiovascular death is more than $50 \%$ higher than the rest of the population. Early myocardial dysfunction in RA patients may be detectable sooner using speckle-tracking echocardiography.

METHOD: Cross-sectional study enrolled 55 patients with RA (mean age 44.1 years) without known cardiovascular disease and 31 healthy controls. All subjects underwent a standard echocardiographic examination: indexed left ventricular mass, left ventricle ejection fraction, isovolumic contraction and relaxation times (IVCT and IVRT), mitral valve inflow curve (E/A), septal mitral annular motion (e'), and E/e' ratio as well as the speckle tracking assessment of left ventricle longitudinal, radial and circular strain and strain rate.

RESULTS: In standard echocardiographic examination RA patients exhibited higher indexed left ventricle mass $\left(96.36 \pm 20.90 \mathrm{~g} / \mathrm{m}^{2}\right.$ vs $\left.95.84 \pm 21.86 \%, \mathrm{p}=0.013\right)$, lower ejection fraction $(64.84 \pm 3.87 \%$ vs $67.10 \pm 3.87 \%, \mathrm{p}=0.011)$ and prolonged IVCT $(61.51 \pm 9.30 \mathrm{~ms}$ vs $53.71 \pm 8.95 \mathrm{~ms}, \mathrm{p}=0.001)$. Diastolic dysfunction was demonstrated by prolonged IVRT $(81.62 \pm 9.56 \mathrm{~ms}$ vs $74.58 \pm 12.02 \mathrm{~ms}, \mathrm{p}=0.007)$ as well as by higher E/e' ratio $(8.21 \pm 1.76$ vs $7.21 \pm 1.52, p=0.009)$. Speckle-tracking method detected lower global longitudinal epicardial strain $(-19.51 \%$ vs $-21.46 \%, p=0.049$ ). Radial, circular, and transversal strains and strain rates were same in both groups. Global longitudinal epicardial strain correlated with IVCT and IVRT, disease duration, and markers of myocardial damage NTproBNP.

CONCLUSIONS: Standard echocardiographic assessment of myocardial function is examiner- and angle-dependent method with considerable limitations for evaluation of minimal subclinical changes. Speckle-tracking echocardiography significantly revealed incipient myocardial dysfunction in RA patients without overt cardiovascular diseases. This correlates with clinical RA characteristics and markers of cardiac damage (Tab. 4, Ref. 48). Text in PDF www.elis.sk.

KEY WORDS: rheumatoid arthritis, left ventricle longitudinal strain, speckle-tracking echocardiography, cardiovascular risk, diastolic dysfunction.
\end{abstract}

\section{Introduction}

Diastolic dysfunction is an early marker of myocardial damage in most commonly encountered cardiovascular diseases. Despite the contradictions about its clinical and prognostic value (1), there is a consensus about its increasing importance in non-cardiac diseases such as rheumatoid arthritis (RA) and other collagen diseases due to early occurrence (2-4).

RA patients experience cardiovascular events earlier (5), have shorter life expectancy (6) and their risk of cardiovascular death is more than $50 \%$ higher (7) than that of the rest of the population. Moreover, these clinical events are only partially dependent

${ }^{1} 5$ th Department of Internal Medicine, Comenius University, Bratislava, Slovakia, ${ }^{2} 2$ nd Department of Internal Medicine, Comenius University, Bratislava, Slovakia, and ${ }^{3}$ Internal Clinic, University of Trnava, Trnava, Slovakia

Address for correspondence: O. Benacka, MD, The National Institute of Cardiovascular Diseases Pod Krasnou horkou 1, SK-831 01 Bratislava, Slovakia.

Phone: +421.905559765, Fax: +421.2.59320.613

Acknowledgement: This work was supported by the Grant of Comenius University (UK/488/2011). on conventional cardiovascular risk factors $(8,9)$ probably due to chronic inflammation (10) and inflammatory myocardial infiltration (11-15). Until now, Doppler detected diastolic dysfunction has been considered to be a typical early marker of myocardial dysfunction in RA patients (16-21). However, Doppler ultrasonography is an angle-dependent method and therefore, the strain and strain rate could be earlier and more accurately evaluated using the speckle-tracking method (22-25).

The aim of this study was to detect subclinical pathologies in myocardial function in RA patients using standard and novel echocardiographic methods, and analyze their relationship to clinical characteristics and laboratory risk markers of RA.

\section{Methods}

Cross-sectional study comprised patients aged 25-55 years with at least 5-year history of seropositive RA without known cardiovascular disease. The study group was recruited from outpatients registers at the 5th and 2nd Departments of Internal Medicine in Bratislava and the Internal Department in Trnava, between 2012 and 2013. 
Exclusion criteria eliminated patients with histories of congestive heart failure, coronary artery disease, atrial fibrillation, stroke, diabetes mellitus, cancer, chronic kidney disease, or venous thromboembolism. The control group was recruited from cohort of 50 employees of participating departments who were matched for age, sex, blood pressure, BMI and smoking habit roughly in ratio 1 control to 2 patients until the both groups had same characteristics.

Both groups underwent structuralized interview, basic anthropometric measurements and blood sampling for classic risk factors and safety parameters. N-terminal prohormone brain natriuretic peptide (NTproBNP) was analyzed as standard marker of myocardial damage. The Health Assessment Questionnaire (HAQ) was used to determine the mobility and functional capacity and the composite index Disease Activity Score (DAS28) was used to determine rheumatoid arthritis inflammatory activity.

Patients and controls underwent an echocardiographic examination using an Esaote MyLab 60 ultrasonograph, which was performed by a single investigator. Left ventricle systolic function was assessed by calculated ejection fraction (Teicholz), isovolumic contraction time (IVCT) and speckle tracking measured systolic strain and strain rate. Diastolic function was assessed by isovolumic relaxation time (IVRT), pulsed-wave Doppler mitral valve inflow curve (E/A), and tissue Doppler imaging (TDI) was used to determine the septal mitral annular motion (e'/a'). The e' wave value (cut-off $\leq 8 \mathrm{~cm} / \mathrm{s}$ ) and the E/e' ratio (cut-off $\leq 9 \mathrm{~cm} / \mathrm{s}$ ) were considered the most sensitive parameters for diastolic dysfunction detection (26). Also, indexed left ventricular mass and aortic root diameters were measured.

By using speckle-tracking echocardiography we estimated longitudinal epicardial, longitudinal endocardial, radial, transversal, circumferential strain (\%) and strain rate (1/s). We measured it separately for six segments in every cross section and then averaged for the global myocardium. Cross-sectioning was done in longitudinal (LAX) and transversal (SAX) planes at the mitral annulus level. In the LAX plane, longitudinal and transversal strain and strain rate were estimated, whereas in the SAX plane, radial and circumferential strain and strain rate were estimated (27-32).

Ethics: The study protocol was approved by the Ethics committee of University Hospital of Medical Faculty of Comenius University and each participant signed an approved the informed consent.

\section{Statistics}

Statistical analyses were performed using SPSS 19.0 software (IBM SPSS Inc., Chicago, Illinois, USA). The impact of clinical characteristics was tested with non-parametric tests of qualitative data ( $\chi 2$ test with Yates's correction or Fisher's exact test as appropriate). Parametric data were first tested for normal distribution using the Kolmogorov-Smirnov test, followed by Student's t-test or ANOVA techniques. The level of statistical significance was conventionally set up at $\mathrm{p}<0.05$.

To quantify the correlation between continuous parameters, Pearson's correlation coefficient was calculated, whereby a value of 1 represents an ideal correlation between two methods. Statistical significance was considered at the level of $p<0.05$. In multiple comparisons (ANOVA), Bonferroni's correction was applied.

\section{Results}

The cross-sectional study included 55 patients with confirmed RA without known cardiovascular disease symptomatic for $13 \pm 8$ years and diagnosed for $11 \pm 7$ years, Control group consisted of 31 healthy persons.

The RA patients were similar to controls in terms of classic risk factors for cardiovascular disease: blood pressure, smoking habit,

Tab. 1. Clinical characteristics of 55 patients with rheumatoid arthritis and 31 healthy controls. Values are mean (SD) unless otherwise noted.

\begin{tabular}{lccc}
\hline Parameter & RA $(\mathrm{n}=55)$ & Control $(\mathrm{n}=31)$ & pa \\
\hline Age $($ years $)$, mean $($ range $)$ & $44.1(36-52)$ & $43.6(34-54)$ & 0.824 \\
\hline Men/women $(\%)$ & $80 / 20$ & $74 / 26$ & 0.777 \\
\hline BMI $\left(\mathrm{kg} / \mathrm{m}^{2}\right)$ & $25.6(5.4)$ & $23.7(4.4)$ & 0.108 \\
\hline Smoking $(\%)$ & 20 & 26 & 0.593 \\
\hline Systolic BP $(\mathrm{mmHg})$ & $128(17)$ & $125(13)$ & 0.478 \\
\hline Diastolic BP $(\mathrm{mmHg})$ & $83(11)$ & $81(10)$ & 0.399 \\
\hline Total cholesterol $(\mathrm{mmol} / \mathrm{l})$ & $5.3(0.9)$ & $5.6(0,9)$ & 0.195 \\
\hline LDL $(\mathrm{mmol} / \mathrm{l})$ & $3.2(0.8)$ & $3.6(0.9)$ & 0.023 \\
\hline Triacylglycerol $(\mathrm{mmol} / \mathrm{l})$ & $1.1(0.6)$ & $1.0(0.5)$ & 0.256 \\
\hline Heart rate $(1 / \mathrm{min})$ & $68.2(9.95)$ & $66.5(11.88)$ & 0.483 \\
\hline WBC $\left(\times 10^{9} / \mathrm{l}\right)$ & $6.9(2.9)$ & $5.7(1.1)$ & $0.006^{* *}$ \\
\hline Haemoglobin $(\mathrm{g} / \mathrm{l})$ & $134(13)$ & $142(14)$ & $0.026^{*}$ \\
\hline GFR-MDRD $(\mathrm{ml} / \mathrm{s})$ & $1.67(0.36)$ & $1.55(0.24)$ & 0.091 \\
\hline NTproBNP $(\mathrm{ng} / \mathrm{l})$ & $80.60(61.88)$ & $51.90(35.50)$ & $0.008^{* *}$ \\
\hline VAS score & $44(21)$ & $10(12)$ & $0.001^{* *}$ \\
\hline HAQ mobility index & 0.97 & - & - \\
\hline DAS28 composite index & $3.4(1.2)$ & $1.0(0.67)$ & $0.001^{* *}$ \\
\hline
\end{tabular}

a Statistical testing done using Student's t-test, BMI - body mass index, BP - blood pressure, LDL - low density lipoproteins; WBC - white blood cells, ESR - erythrocyte sedimentation rate, GFR-MDRD - glomerular filtration rate by MDRD equation, NTproBNP - N-terminal of the prohormone brain natriuretic peptide, VAS - visual analogue scale, HAQ - Health Assessment Questionnaire, DAS - Disease Activity Score

Tab. 2. Echocardiographic results. Values are means (SD) unless otherwise noted.

\begin{tabular}{lccc}
\hline Parameter & RA $(\mathrm{n}=55)$ & Control $(\mathrm{n}=31)$ & $\mathrm{p}^{\mathrm{a}}$ \\
\hline LVEDD $(\mathrm{mm})$ & $47.03(4.65)$ & $45.44(3.74)$ & 0.088 \\
\hline LVMI $\left(\mathrm{g} / \mathrm{m}^{2}\right)$ & $96.36(20.90)$ & $95,84(21.86)$ & $0.013^{*}$ \\
\hline Left atrium $(\mathrm{mm})$ & $30.02(3.52)$ & $29.10(3.61)$ & 0.252 \\
\hline Aortic annulus $(\mathrm{mm})$ & $29.31(3.44)$ & $25.84(3.63)$ & $0.001^{* *}$ \\
\hline Sinuses of Valsalva $(\mathrm{mm})$ & $33.16(3.53)$ & $30.06(3.75)$ & $0.001^{* *}$ \\
\hline EF Teicholz $(\%)$ & $64.84(3,87)$ & $67.10(3,87)$ & $0.011^{*}$ \\
\hline IVCT $(\mathrm{ms})$ & $61.51(9.30)$ & $53.71(8.95)$ & $0.001^{* *}$ \\
\hline IVRT $(\mathrm{ms})$ & $81.62(9.56)$ & $74.58(12.02)$ & $0.007^{* *}$ \\
\hline E wave $(\mathrm{cm} / \mathrm{s})$ & $74.02(13.70)$ & $71.94(17.53)$ & 0.543 \\
\hline A wave $(\mathrm{cm} / \mathrm{s})$ & $61.78(16.09)$ & $55.26(13.13)$ & 0.058 \\
\hline E/A & $1.27(0.38)$ & $1.37(0.51)$ & 0.287 \\
\hline e'wave $(\mathrm{cm} / \mathrm{s})$ & $9.47(1.83)$ & $10.06(2.06)$ & 0.174 \\
\hline a'wave $(\mathrm{cm} / \mathrm{s})$ & $7.62(1.82)$ & $7.55(1.69)$ & 0.861 \\
\hline e'/a' & $1.30(0.42)$ & $1.45(0.58)$ & 0.226 \\
\hline E/e' mean & $8.21(1.76)$ & $7.21(1.52)$ & $0.009 * *$
\end{tabular}

a Statistical testing done using Student's t-test, LVEDD - left ventricle end-diastolic diameter, LVMI - left ventricle mass/indexed, EF - ejection fraction calculated with Teicholz formula, IVCT - isovolumic contraction time, IVRT - isovolumic relaxation time 
Tab. 3. Strain and strain rate (segmental and overall), determined by the speckle-tracking method. Values are means.

\begin{tabular}{|c|c|c|c|c|c|c|c|}
\hline Parameter & RA & Control & $\mathrm{p}^{\mathrm{a}}$ & Parameter & RA & Control & $\mathrm{p}^{\mathrm{a}}$ \\
\hline SLonEndoBS & -17.28 & -18.96 & 0.280 & SRLonEndoBS & -1.45 & -1.46 & 0.953 \\
\hline SLonEndoMS & -20.62 & -22.13 & 0.178 & SRLonEndoMS & -1.30 & -1.38 & 0.239 \\
\hline SLonEndoAS & -26.22 & -27.76 & 0.317 & SRLonEndoAS & -1.54 & -1.73 & 0.195 \\
\hline SLonEndoAL & -21.15 & -23.56 & 0.112 & SRLonEndoAL & -1.37 & -1.37 & 0.251 \\
\hline SLonEndoML & -19.95 & -20.96 & 0.405 & SRLonEndoML & -1.37 & -1.52 & 0.07 \\
\hline SLonEndoBL & -21.10 & -21.67 & 0.691 & SRLonEndoBL & -1.61 & -1.77 & 0.226 \\
\hline SLonEndoAve & -21.05 & -22.51 & 0.119 & SRLonEndoAve & -1.43 & -1.55 & 0.072 \\
\hline SLonEpiBS & -24.54 & -26.73 & 0.131 & SRLonEpiBS & -1.72 & -1.80 & 0.523 \\
\hline SLonEpiMS & -19.77 & -21.30 & 0.135 & SRLonEpiMS & -1.20 & -1.31 & 0.17 \\
\hline SLonEpiAS & -12.15 & -13.49 & 0.083 & SRLonEpiAS & -0.74 & -0.81 & 0.145 \\
\hline SLonEpiAL & -12.92 & -15.49 & 0.004 ** & SRLonEpiAL & -0.84 & -0.88 & 0.587 \\
\hline SLonEpiML & -21.73 & -23.99 & 0.099 & SRLonEpiML & -1.46 & -1.62 & 0.145 \\
\hline SLonEpiBL & -25.92 & -27.73 & 0.282 & SRLonEpiBL & -1.88 & -1.86 & 0.907 \\
\hline SLonEpiAve & -19.51 & -21.46 & 0.049 * & SRLonEpiAve & -1.30 & -1.38 & 0.349 \\
\hline
\end{tabular}

Tab. 4. Correlation ( $r, p$ values) between clinical variables and parameters of left ventricular functions.

\begin{tabular}{|c|c|c|c|c|c|c|c|c|}
\hline \multirow{2}{*}{ RA patients } & \multicolumn{2}{|c|}{ SLonEndo } & \multicolumn{2}{|c|}{ SRLonEndo } & \multicolumn{2}{|c|}{ SLongEpi } & \multicolumn{2}{|c|}{ SRLongEpi } \\
\hline & $\mathrm{r}$ & $p$ & $r$ & $p$ & $\mathrm{r}$ & $\mathrm{p}$ & $\mathrm{r}$ & $p$ \\
\hline DAS28 & 0.232 & 0.089 & 0.176 & 0.198 & 0.066 & 0.635 & 0.071 & 0.605 \\
\hline HAQ & -0.085 & 0.539 & -0.206 & 0.132 & -0.032 & 0.817 & -0.189 & 0.167 \\
\hline VAS & 0.104 & 0.451 & -0.032 & 0.817 & 0.083 & 0.546 & -0.059 & 0.670 \\
\hline RA duration & $-0,055$ & 0.691 & -0.048 & 0.726 & -0.272 & $0.044 *$ & -0.146 & 0.287 \\
\hline NTproBNP & -0.103 & 0.456 & -0.228 & 0.095 & -0.176 & 0.199 & $-0.277 *$ & 0.041 * \\
\hline $\mathrm{EF}$ & -0.111 & 0.419 & 0.094 & 0.496 & -0.128 & 0.351 & -0.15 & 0.274 \\
\hline IVRT & 0.214 & 0.117 & $0.293 *$ & $0.030 *$ & 0.129 & 0.346 & 0.098 & 0.474 \\
\hline E/e' & 0.217 & 0.112 & $0.299 *$ & 0.026 * & 0.19 & 0.165 & 0.35 & 0.084 \\
\hline \multicolumn{9}{|l|}{ Controls } \\
\hline NTproBNP & -0.154 & 0.408 & -0.081 & 0.665 & -0.181 & 0.331 & -0.099 & 0.597 \\
\hline $\mathrm{EF}$ & -0.285 & 0.120 & -0.017 & 0.928 & -0.177 & 0.342 & -0.01 & 0.956 \\
\hline IVRT & 0.092 & 0.622 & 0.103 & 0.582 & 0.146 & 0.432 & 0.057 & 0.760 \\
\hline$E / e^{6}$ & -0.306 & 0.094 & -0.065 & 0.728 & -0.131 & 0.482 & -0.016 & 0.930 \\
\hline
\end{tabular}

Statistical testing done using, "regular font" - Spearman's rank correlation coefficient, "italic font" - Pearson's correlation coefficient, BMI - body mass index, BP - blood pressure, LDL - low density lipoproteins, WBC - white blood cells, ESR - erythrocyte sedimentation rate, GFR-MDRD - glomerular filtration rate by MDRD equation, DAS - Disease Activity Score, HAQ - Health Assessment Questionnaire, VAS - visual analogue scale, RA - rheumatoid arthritis, NTproBNP - N-terminal of the prohormone brain natriuretic peptide, EF - ejection fraction, IVRT - isovolumic relaxation time, E - transmitral Doppler flow E (early) wave, e' - tissue Doppler septal early wave

glycaemia, total cholesterol and triacylglycerol levels (Tab. 1). No significant difference between groups was found in laboratory parameters, except for explainable higher leucocyte level and lower hemoglobin level in RA patients. The NTproBNP level was higher in RA patients than in controls $(80.60 \pm 61.88 \mathrm{ng} / \mathrm{l}$ vs $51.90 \pm 35.50 \mathrm{ng} / \mathrm{l}, \mathrm{p}=0.008)$, and exhibited weak correlation with the HAQ mobility index $(\mathrm{r}=0.318, \mathrm{p}=0.018)$ and with disease duration $(\mathrm{r}=0.261, \mathrm{p}=0.054)$.

In echocardiographic examination, RA patients had more extended sinuses of Valsalva $(33.16 \pm 3.53 \mathrm{~mm}$ vs $30.06 \pm 3.75 \mathrm{~mm}$, $\mathrm{p}=0.001)$ and a larger aortic annular base diameter $(29.31 \pm 3.44$ $\mathrm{mm}$ vs $25.84 \pm 3.63 \mathrm{~mm}, \mathrm{p}=0.001$ ) as compared to controls.

As related to diastolic dysfunction, RA patients showed a higher E/e' ratio ( $8.21 \pm 1.76$ vs $7.21 \pm 1.52$, $\mathrm{p}=0.009)$, but the mean values of e' did not differ between groups. IVRT was prolonged in RA patients in comparison with controls $(81.62 \pm 9.56 \mathrm{~ms}$ vs $74.58 \pm 12.02 \mathrm{~ms}, \mathrm{p}=0.007$ ).
Regarding systolic function, RA patients had significantly prolonged IVCT $(61.51 \pm 9.30 \mathrm{~ms}$ vs $53.71 \pm 8.95 \mathrm{~ms}, \mathrm{p}=0.001)$ and decreased EF $(64.84 \pm 3.87 \%$ vs $67.10 \pm 3.87 \%, \mathrm{p}=0.011)$ than the controls (Tab. 2). Speckle-tracking assessment of left ventricular function revealed decreased global longitudinal epicardial strain $(-19.51 \%$ vs $-21.46 \%, p=0.049)$, and decreased local longitudinal epicardial strain in apicolateral segment $(-12.92 \% \mathrm{vs}-15.49 \%$, $\mathrm{p}=0.004$ ) (Tab. 3). Radial, transversal and circumferential strain analysis did not exhibit significant differences.

Correlation analysis of strain parameters with echocardiographic parameters showed direct correlation of longitudinal endocardial strain rate values with IVRT $(r=0.29 ; \mathrm{p}=0.030)$ and $\mathrm{E} / \mathrm{e}$ ' ratio $(\mathrm{r}=0.30 ; \mathrm{p}=0.026)$. As related to clinical characteristics and markers, indirect correlations were found between longitudinal epicardial strain rate and the NTproBNP level $(\mathrm{r}=0.28 ; \mathrm{p}=0.041)$. Global longitudinal epicardial strain was indirectly correlated with RA disease duration $(r=0.28 ; \mathrm{p}=0.044)$ (Tab. 4). 


\section{Discussion}

The comparison of standard echocardiographic and Doppler parameters as age, gender, BMI and smoking habits matched RA patients without known cardiovascular disease with controls and did not reveal dramatic differences in myocardial structure and function, thus confirming difficulties in identification of early myocardial abnormality markers for high risk in RA patients. Majority of the differences between groups were within the reference values for given parameters, including even the contrast differences in NTproBNP, considered standard for early detection of myocardial dysfunction. Identification of causal relationship between echocardiographical parameters with soluble biomarkers and clinical characteristics could be therefore dependent on modulating factors as age, duration of disease, functional capacity, therapy, concomitant disease and other difficult-to-balance factors.

NTproBNP is a cardiac neurohormone responding to ventricular overload, hypoxia and increased ventricular wall tension or hypertrophy, and in RA patients correlates with systolic and diastolic volumes and left ventricular mass as measured by Doppler echocardiography (33-36). In our study with participants balanced for blood pressure and other traditional risk factors, we did not detect a correlation between the NTproBNP level and standard echocardiography parameters for systolic (EF, IVCT) and diastolic function (IVRT, E/e'), similarly to previous studies patients with chronic inflammatory disease $(37,38)$. Both LV mass or volumic overload parameters did not explain differences in NTproBNP between RA patients and controls.

Our RA patients had a significantly prolonged IVRT, which may indicate some active relaxation disturbance of the left ventricle in early diastole, as already suggested by Lascano et al (39) and other authors $(40,41)$ and it may tempt to explain the increase in NTproBNP. However, the correlation is only borderline $(r=0.35$; $\mathrm{p}=0.054)$. Any of mentioned studies, including our own, did not find correlation between IVRT with clinical characteristics as disease duration.

However, a significant correlation was found between the NTproBNP level and myocardial function assessed by speckletracking echocardiography. The dominant speckle-tracking parameter is considered to be the longitudinal strain and strain rate $(29,32,42)$. Our study demonstrated lower trend for the average values of longitudinal strain and strain rate in RA patients than in healthy controls in both global and defined segments of the left ventricle, predominantly affecting apicolateral epicardial segment.

Recent study by Midtbo (43) showed that speckle tracking can be a sensitive method for finding subclinical left ventricle dysfunction. Also, the study by Fine et al (23) confirmed a decreased global left ventricle longitudinal strain in RA patients and its correlations with a reduced HAQ mobility index, and contrary to our patients, a lack of correlation with disease duration. Longitudinal strain in this study might have been influenced by more prevalent classic cardiovascular risk factors (hypertension $85 \%$; smoking habit $44 \%$; and dyslipidemia $71 \%$, diabetes mellitus $14 \%$ ) as well as higher blood pressure in RA patients. More reduced HAQ mobi- lity index and lack of correlation with disease duration in Fine et al study could be explained by higher age ( 55.7 vs 44.1 years). However, presented study had these risk factors strictly balanced and provided the same result suggesting the causal effect of RA on longitudinal strain.

The association between impaired strain parameters and duration of RA was found also by other authors. In a small study of Sitia et al (22 patients and 20 controls) which used similar inclusion and exclusion criteria as in our study, RA patients exhibited similar abnormalities in longitudinal strain correlating with disease duration (22). Baktir et al compared 37 RA patients divided in two age-matched groups (45 years) according to disease duration (2.8 vs 14.6 years; no healthy control group) and found significantly lower longitudinal strain parameters in patients with advanced disease (24). Both studies did not find any correlation between radial and circumferential strain parameters.

Consistent relationship of londitudinal strain parameters with biomarkers and clinical characteristics implicate causal involvement of anterior epicardial segment in early stage of RA associated with other early markers of LV dysfunction (22-24).

In patients with diastolic dysfunction, arterial hypertension is frequently diagnosed with the prevalence of $60-88 \%(44,45)$. Majority of cited RA studies $(8,10,11,13)$ studied groups with comparable blood pressure and therefore, the slightly impaired diastolic parameters and LV mass in RA patients cannot be explained by the effect of hypertension on diastolic function (46).

Major limitation of presented study could be the lackof interobserver comparison of speckle-tracking measurement which can cause "virtual dyskinesia" due to intraindividual error (47, 48). When the endocardial borders are manually traced even by experienced specialist, subjective evaluation may overestimate the myocardial thickness and lead to impaired epicardial strain assessment. The measurement of total and segmental strain could be improved by the measurement of isovolumic times, maximum mitral inflow velocities, and mitral annulus motion which decrease the chance of false-positive strain assessment.

On other side, even when reproducibility of the strain measurements is conditioned by inter-individual variability, studies with one investigator $(22,25)$ as well as studies with more operators $(23,24)$ obtained relatively consistent findings.

\section{Conclusion}

Standard echocardiographic assessment of myocardial functions in patients with RA without signs of cardiovascular disease suggested minimal changes in left ventricular systolic function (by IVCT and LVEF by Teicholz) and diastolic function (by E/e' and IVRT).

The speckle-tracking method for myocardial strain analysis showed an unambiguous impairment of longitudinal strain parameters, which correlates with biomarkers and clinical characteristics of RA. Speckle-tracking method has the advantage of being independent of preload and afterload, as well as insonation angle. Due to its sensitivity and continuous improvements in software packages, especially for the three dimensional evalu- 
ation of left ventricular strain, the method may be an appropriate for early detection of subclinical myocardial impairment in rheumatic patients.

\section{References}

1. Abhayaratna WP et al. Characteristics of left ventricular diastolic dysfunction in the community: an echocardiographic survey. Heart 2006; 92 (9): 1259-1264.

2. Vizzardi $\mathbf{E}$ et al. Echocardiographic evaluation of asymptomatic patients affected by rheumatoid arthritis. J Invest Med 2012; 60 (8): 1204-1208.

3. Bayram NA et al. Assessment of left ventricular functions in patients with Sjögren's syndrome using tissue Doppler echocardiography. Intern J Rheum Dis 2013; 16 (4): 425-429.

4. Sharma A et al. Echocardiographic evaluation of diastolic dysfunction in rheumatoid arthritis - a case-control study. Modern Rheumatol 2015; 25 (4): 552-557.

5. Peters $\mathbf{M}$ et al. EULAR evidence-based recommendations for cardiovascular risk management in patients with rheumatoid arthritis and other forms of inflammatory arthritis. Ann Rheum J 2010; 69 (2): 325-331.

6. Wong JB, Ramey DR, Singh G. Long-term morbidity, mortality, and economics of rheumatoid arthritis. Arthritis Rheum 2001; 44 (12): 27462749.

7. Aviña-Zubieta JA et al. Risk of cardiovascular mortality in patients with rheumatoid arthritis: A meta-analysis of observational studies. Arthritis Care Res 2008; 59 (12): 1690-1697.

8. deL Rincon I et al. High incidence of cardiovascular events in a rheumatoid arthritis cohort not explained by traditional cardiac risk factors. Arthritis Rheum 2001; 44 (12): 2737-2745.

9. Roman MJ et al. Preclinical carotid atherosclerosis in patients with rheumatoid arthritis. Ann Intern Med 2006; 144 (4): 249-256.

10. Solomon DH et al. Explaining the cardiovascular risk associated with rheumatoid arthritis: traditional risk factors versus markers of rheumatoid arthritis severity. Ann Rheum Dis 2010.

11. Radovits BJ et al. Disease activity as a risk factor for myocardial infarction in rheumatoid arthritis. Ann Rheum Dis 2009; 68 (8): 1271-1276.

12. Rovenský J, Vlcek M, Imrich R. Cardiovascular diseases in rheumatoid arthritis). Vnitr Lek 2010; 56 (7): 721-723.

13. Innala $\mathbf{L}$ et al. Cardiovascular events in early RA are a result of inflammatory burden and traditional risk factors: a five year prospective study. Arthritis Res Ther 2011; 13 (4): 1.

14. Karpouzas GA et al. Prevalence, extent and composition of coronary plaque in patients with rheumatoid arthritis without symptoms or prior diagnosis of coronary artery disease. Ann Rheum Dis 2014; 73 (10): 1797-1804.

15. Myasoedova E et al. Brief Report: Rheumatoid Arthritis Is Associated With Left Ventricular Concentric Remodeling: Results of a PopulationBased Cross-Sectional Study. Arthritis Rheum 2013; 65 (7): 1713-1718.

16. Corrao $S$ et al. Echo-Doppler left ventricular filling abnormalities in patients with rheumatoid arthritis without clinically evident cardiovascular disease. Eur J Clin Invest 1996; 26 (4): 293-297.

17. Di Franco M et al. Diastolic function abnormalities in rheumatoid arthritis. Evaluation by echo Doppler transmitral flow and pulmonary venous flow: relation with duration of disease. Ann Rheum Dis 2000; 59 (3): $227-229$
18. Alpaslan M, Onrat E, Evcik D. Doppler echocardiographic evaluation of ventricular function in patients with rheumatoid arthritis. Clin Rheumatol 2003; 22 (2): 84-88.

19. Rexhepaj $\mathbf{N}$ et al. Left and right ventricular diastolic functions in patients with rheumatoid arthritis without clinically evident cardiovascular disease. Internat J Clin Pract 2006; 60 (6): 683-688.

20. Meune $\mathbf{C}$ et al. Myocardial dysfunction in rheumatoid arthritis: a controlled tissue - Doppler echocardiography study. J Rheumatol 2007; 34 (10): 2005-2009.

21. Yazici $D$ et al. Echocardiographic evaluation of cardiac diastolic function in patients with rheumatoid arthritis: 5 years of follow-up. Clin Rheumatol 2008; 27 (5): 647-650.

22. Sitia $S$ et al. Detection of preclinical impairment of myocardial function in rheumatoid arthritis patients with short disease duration by speckle tracking echocardiography. Internat J Cardiol 2012; 160 (1): 8-14.

23. Fine NM et al. Evaluation of myocardial function in patients with rheumatoid arthritis using strain imaging by speckle-tracking echocardiography. Ann Rheum Dis 2014; 73 (10): 1833-1839.

24. Baktir A et al. Preclinical impairment of myocardial function in rheumatoid arthritis patients. Herz 2015; 40 (4): 669-674.

25. Logstrup BB et al. Left ventricular function in treatment-naive early rheumatoid arthritis. Amer J Cardiovasc Dis 2014; 4 (2): 79.

26. Nagueh SF et al. Recommendations for the evaluation of left ventricular diastolic function by echocardiography. J Amer Soc Echocardiogr 2009; 22 (2): 107-133.

27. Geyer $\mathbf{H}$ et al. Assessment of myocardial mechanics using speckle tracking echocardiography: fundamentals and clinical applications. J Amer Soc Echocardiogr 2010; 23 (4): 351-369.

28. Mor-Avi V et al. Current and evolving echocardiographic techniques for the quantitative evaluation of cardiac mechanics: ASE/EAE consensus statement on methodology and indications: endorsed by the Japanese Society of Echocardiography. J Amer Soc Echocardiogr 2011; 24 (3): 277-313.

29. Buccheri S et al. Feasibility, reproducibility, and agreement between different speckle tracking echocardiographic techniques for the assessment of longitudinal deformation. BioMed Res Internat 2013; 2013.

30. Chapman CB et al. Classification of left ventricular diastolic function using American Society of Echocardiography Guidelines: agreement among echocardiographers. Echocardiography 2013; 30 (9): 1022-1031.

31. Abduch MCD et al. Cardiac mechanics evaluated by speckle tracking echocardiography. Arq Brasil Cardiol 2014; 102 (4): 403-412.

32. Mada RO, Duchenne J, Voigt JU. Tissue Doppler, Strain and Strain Rate in ischemic heart disease "How I do it". Cardiovasc Ultrasound 2014; 12 (1): 1 .

33. Kozera L, Andrews J, Morgan AW. Cardiovascular risk and rheumatoid arthritis - the next step: differentiating true soluble biomarkers of cardiovascular risk from surrogate measures of inflammation. Rheumatology 2011; 50 (11): 1944-1954.

34. Harney $S$ et al. Brain natriuretic peptide is a potentially useful screening tool for the detection of cardiovascular disease in patients with rheumatoid arthritis. Ann Rheum Dis 2006; 65 (1): 136.

35. Provan SA et al. The association between disease activity and NTproBNP in 238 patients with rheumatoid arthritis: a 10-year longitudinal study. Arthritis Res Ther 2008; 10 (3): 1. 
36. Provan S et al. NT-proBNP predicts mortality in patients with rheumatoid arthritis: results from 10-year follow-up of the EURIDISS study. Annm Rheum Dis 2010: annrheumdis127704.

37. George J, Khan F, Struthers A. The source of BNP in rheumatoid arthritis. Internat J Cardiol 2014; 174 (3): 740.

38. Tomáš L' et al. Acute and long-term effect of infliximab on humoral and echocardiographic parameters in patients with chronic inflammatory diseases. Clin Rheumatol 2013; 32 (1): 61-66.

39. Lascano $\mathbf{C}$ et al. (Ventricular diastolic dysfunction in rheumatoid arthritis). Revista de la Facultad de Ciencias Medicas (Cordoba, Argentina) 2008; 66 (2): 58-65.

40. Levendoglu $\mathbf{F}$ et al. Ventricular function abnormalities in active rheumatoid arthritis: a Doppler echocardiographic study. Rheumatol Internat 2004; 24 (3): 141-146.

41. Udayakumar N, Venkatesan S, Rajendiran C. Diastolic function abnormalities in rheumatoid arthritis: relation with duration of disease. Singapore Med J 2007; 48 (6): 537.

42. Ernande $L$ et al. Impaired myocardial radial function in asymptomatic patients with type 2 diabetes mellitus: a speckle-tracking imaging study. J Amer Soc Echocardiogr 2010; 23 (12): 1266-1272.
43. Midtbo $\mathbf{H}$ et al. Disease activity is associated with reduced left ventricular systolic myocardial function in patients with rheumatoid arthritis. Ann Rheum Dis 2016: annrheumdis-2016-209223.

44. McMurray JJ et al. Heart failure with preserved ejection fraction: Clinical characteristics of 4133 patients enrolled in the I-PRESERVE trial. Eur J Heart Failure 2008; 10 (2): 149-156.

45. Yusuf $S$ et al. Effects of candesartan in patients with chronic heart failure and preserved left-ventricular ejection fraction: the CHARM-Preserved Trial. Lancet 2003; 362 (9386): 777-781.

46. Corrao $\mathbf{S}$ et al. Rheumatoid arthritis affects left ventricular mass: Systematic review and meta-analysis. Eur J Intern Med 2015; 26 (4): 259-267.

47. Kumar V et al. Strain Rate Imaging: Real World Scenario? J Cardiovasc Dis Diagn 2014; 2014.

48. Arias-Godínez JA et al. Function and mechanics of the left ventricle: from tissue Doppler imaging to three dimensional speckle tracking. Arch Cardiol Mex 2011; 81 (2): 114-125.

Received September 21, 2016. Accepted October 11, 2016 